\title{
Numerical Optimization of Pressure Force from Multiple Jets Impinging on a Moving Curved Surface for Industrial Drying Machines
}

\author{
Ali Chitsazan ${ }^{1}$, Georg Klepp ${ }^{1}$, Mohammad Esmaeil Chitsazan², Birgit Glasmacher ${ }^{3}$ \\ ${ }^{1}$ Institute for Energy Research, Ostwestfalen-Lippe University of Applied Sciences and Arts, Lemgo, Germany \\ ${ }^{2}$ Research Institute of Education, Ahvaz, Iran \\ ${ }^{3}$ Institute for Multiphase Processes, Leibniz University Hannover, Hannover, Germany \\ Email: *ali.chitsazan@yahoo.com
}

How to cite this paper: Chitsazan, A., Klepp, G., Chitsazan, M.E. and Glasmacher, B. (2021) Numerical Optimization of Pressure Force from Multiple Jets Impinging on a Moving Curved Surface for Industrial Drying Machines. Open Journal of Fluid Dynamics, 11, 192-209.

https://doi.org/10.4236/ojfd.2021.114012

Received: May 22, 2021

Accepted: December 6, 2021

Published: December 9, 2021

Copyright $\odot 2021$ by author(s) and Scientific Research Publishing Inc. This work is licensed under the Creative Commons Attribution International License (CC BY 4.0).

http://creativecommons.org/licenses/by/4.0/

\begin{abstract}
Jet force on the surface is typical for impinging jets towards the surface and it is very important in drying applications for force-sensitive surfaces. The designer should optimize the design parameters of industrial drying equipment to achieve minimum pressure force between multiple jets and a moving curved surface. SST $k$ - $\omega$ turbulence model is used to simulate a real geometry for industrial drying applications. The SST $k-\omega$ turbulence model succeeded with reasonable accuracy in reproducing the experimental results. The jet to surface distance, jet to jet spacing, jet inlet velocity, jet angle, and surface velocity are chosen as the design parameters. For the optimization of the impinging round jet, the pressure force coefficient on the moving curved surface is set as the objective function to be minimized. The SHERPA search algorithm is used to search for the optimal point from the weighted sum of all objectives method. One correlation is developed and validated for the pressure force coefficient. It is found that the pressure force coefficient is highly dependent on the nozzle to surface distance and jet angle but relatively insensitive to jet inlet velocity, jet to jet spacing, and surface velocity. The minimum pressure force coefficient correlates with a high value of nozzle to surface distance (tenfold diameter in this analysis) and a low value of the jet angle $\left(40^{\circ}\right.$ in this analysis). The agreement in the prediction of the pressure force coefficient between the numerical simulation and developed correlation is found to be reasonable and all the data points deviate from the correlation approximately $8 \%$ on average.
\end{abstract}

\section{Keywords}

Multiple Jets, Pressure Force, Surface Motion, Surface Curvature, CFD, 
Optimization

\section{Introduction}

Jet impingements enhance the heat transfer rate in many industrial applications such as cooling, heating, and drying due to the large amounts of heat and mass transfer between the target surface and the working fluid. A large portion of reported works focus on the heat transfer properties and velocity/pressure fields of impinging jet flows [1] [2] [3].

Jet force on the surface is typical for impinging jets towards the surface and it is very important in drying applications for force sensitive products (i.e. paper, fabrics) or force sensitive surfaces (i.e. painted, coated). The nozzle exit velocity could be limited if the product is sensitive to deformation under the jet impinging force. The designer should optimize the design parameters of industrial drying machines to achieve the minimum pressure force on force-sensitive products or surfaces. Nevertheless, this aspect is often omitted in the reported works.

Penumadu and Rao [4] showed that the heat transfer in contrast to the pressure drop is extremely sensitive to minor changes in $H / d$ but is insensitive to manufacturing tolerances in jet diameter. Hebert et al. [5] compared the results for converging and diverging channels with results for parallel plate channels with different spacing to compare the effect of the streamwise pressure gradient. The parallel channels with even spacing throughout show similar trends where the converging channels match in dimension. Similarly, for the diverging channels, this trend is evident. El-Gabry et al. [6] suggested that the use of rough surfaces could enhance the heat transfer significantly and reduce the thermal nonuniformity at negligible additional pressure drop. Kamal et al. [7] stated that the optimum case which satisfies the largest drying rates beside the uniform pressure distributions along the drying plane is $S / d=3.5, H / d=6$, and $\theta=60^{\circ}$. Harrington et al. [8] concluded that the target wall curvature does not have a significant effect in altering the pressure drop from jet array impingement and thus the flow distribution in the channel. Levy et al. [9] found that most of the pressure drop occurs due to the loss of the kinetic energy in the jets. The pressure drop increases rapidly with an increase in the jet Reynolds number and the effect of the nozzle to surface distance on the pressure drop characteristics was found to be low.

Kastner et al. [10] calculated the jet impingement force through the integration of the measured pressure distributions on flat plates due to an impinging jet. Wang et al. [11] found that the mean impact force coefficients from a single circular jet impinging normally onto a fixed flat surface are highly dependent on the nozzle to surface distance, but relatively insensitive to the jet Reynolds number. Page et al. [12] deduced experimentally that wall pressure distribution, reattachment angle, and reattachment radius of radial jet flow are independent of 
the jet Reynolds number. A dimensionless force coefficient has been defined [13] to describe the net surface force of radial jet flows. The radial jet nozzles with flow exit angles of $-10^{\circ}$ in paper machines increase the sheet stability owing to the suction forces [14] [15]. Peper et al. [16] found that the total force exerted by radial jets on a plane surface is lower than that exerted by inline jets and the total force exerted by radial jets decreases with decreasing the flow exit angle.

The pressure force between multiple jets and a moving curved surface is more difficult to study due to the changing boundaries and effect of surface curvature but is also very relevant in engineering applications such as paper drying machines. Only a few experimental studies exist for the impact of the pressure force of a single impinging jet on a fixed flat surface and the data available for multiple jets in the literature is very scarce and the majority of works focus on the heat transfer properties and velocity/pressure fields. Hence, further experimental and numerical investigation of the pressure force in systems of multiple impinging jets on a moving curved surface is necessary to have useful correlations for design purposes and to make the dryer more efficient in terms of flow patterns.

The scope of this research is to find the optimum value of key design parameters of paper drying machines such as the jet to surface distance, jet to jet spacing, jet inlet velocity, jet angle, and surface velocity. The pressure force from multiple jets impinging on the moving curved surface is set as the objective function to be minimized. The weighted sum of all objectives method and the SHERPA search algorithm is used for the optimization study. In an optimization study, the objective is evaluated with respect to the design variables using a CFD model. The CFD model is based on the solution of the stationary Reynolds-averaged Navier-Stokes equation with a finite volume method. Finally, one correlation is developed and validated for the pressure force.

\section{Mathematical Formulation}

In the following, the conservation laws of mass, momentum, and energy are expressed for an incompressible fluid with the constant fluid properties in steady state form:

$$
\begin{gathered}
\frac{\partial U_{i}}{\partial X_{i}}=0 \\
U_{j} \frac{\partial U_{i}}{\partial X_{j}}=\frac{\partial}{\partial X_{j}}\left(v \frac{\partial U_{i}}{\partial X_{j}}\right)-\frac{1}{\rho} \frac{\partial P}{\partial X_{j}} \\
U_{j} \frac{\partial \Theta}{\partial X_{j}}=\frac{\partial}{\partial X_{j}}\left(\Gamma_{\Theta} \frac{\partial \Theta}{\partial X_{j}}\right)+q_{\Theta}
\end{gathered}
$$

The Reynolds-Averaged Navier Stokes equations are solved for the transport of mean flow quantities with appropriate RANS turbulence models to describe the influence of the turbulent quantities to provide closure relations. Each solution variable in the instantaneous Navier-Stokes equations should be decomposed into an averaged value and a fluctuating component to obtain the Rey- 
nolds-Averaged Navier-Stokes equations. The resulting equations for the mean quantities are essentially identical to the original equations, except that an additional term now appears in the momentum transport equation. This additional term, known as the Reynolds stress tensor, has the following definition:

$$
T_{t}=-\overline{U_{i}^{\prime} U_{j}^{\prime}}
$$

The challenge is thus to model the Reynolds stress tensor to close the timeaveraged equations. Eddy viscosity models employ the concept of a turbulent viscosity for modeling of Reynolds stress tensor. The most common model is known as the Boussinesq approximation:

$$
T_{t}=2 v_{t} S_{i j}-\frac{2}{3} \delta_{i j} k
$$

where $v_{t}$ is the turbulent viscosity, $k$ is the turbulence kinetic energy, $\delta_{i j}$ is the Kronecker delta $(=1$ if $i=j$, otherwise $=0)$ and $S_{i j}$ is mean strain rate tensor and given by:

$$
S_{i j}=\frac{1}{2}\left(\frac{\partial \bar{U}_{i}}{\partial X_{j}}+\frac{\partial \bar{U}_{j}}{\partial X_{i}}\right)
$$

Since the assumption that the Reynolds stress tensor is linearly proportional to the mean strain rate and does not consider the anisotropy of turbulence, some two-equation models extend the linear approximation to include the non-linear constitutive relations. The use of hybrid models as a combination of efficient two-equation models is advisable. The Shear Stress Transport (SST) $k-\omega$ model as a combination of the $k-\mathcal{E}$ model in the freestream and the standard $k-\omega$ model in the inner parts of the boundary layer is an obvious choice.

\section{Definition of Characteristic Numbers}

The local heat transfer coefficient is non-dimensionalized to the Nusselt number by the following expression:

$$
N u=\frac{h d}{k}=\frac{q}{T_{w}-T_{j}} \cdot \frac{d}{k}
$$

where $q$ is the convective heat flux, $T_{w}$ is the target wall temperature, $T_{j}$ is the jet exit temperature, $d$ is the jet exit diameter, $k$ is the thermal conductivity of the air at jet exit temperature and $h$ is heat transfer coefficient.

Pressure force on the surface is the force that the fluid exerts in the direction of normal to the surface. Pressure force on the impingement surface is presented in dimensionless form by a force coefficient $C_{f}$ The force coefficient of a surface is defined as follows:

$$
C_{f}=\frac{F}{0.5 \rho V^{2}\left(\pi d^{2} / 4\right)}
$$

where $F$ is the pressure force on the surface, $\rho$ is the density of the fluid, $d$ is the 
diameter of the nozzle and $V$ is the jet exit velocity. The pressure force on the surface is computed as:

$$
F=P_{s t} A
$$

where $P_{s t}$ is the pressure at the stagnation point and $A$ is the target surface area.

\section{Pre-Processing of Simulation Analysis}

Figure 1 shows the geometry of the impinging jet and boundary condition used in the present work. All jet inlets were modeled as circular planes in the top wall. The incoming flow is assumed to be with constant fluid properties at $T=298.15$ ${ }^{\circ} \mathrm{K}$, entered with a uniform velocity profile. The value of inlet velocity is selected so that it matches with the Reynolds number over the range of $4337-21,685$. The target surface i.e. a moving curved surface was modeled as a no-slip wall held at a constant temperature of $T_{W}=60^{\circ} \mathrm{C}$. On all other solid surfaces, a no-slip and adiabatic wall boundary condition is imposed. A constant pressure outlet boundary condition is applied to all open boundaries. The movement of the curved surface is considered along curvilinear axes. A symmetric boundary condition was also applied in the $\mathrm{X}-\mathrm{Y}$ plane for the central jet to reduce the computational cost.

The numerical model is based on the solution of the stationary Reynoldsaveraged Navier-Stokes equation with a finite volume method. The CFD model is set up and run with the commercial code STAR-CCM ${ }^{+} 13.02 .013$ by CD-Adapco.

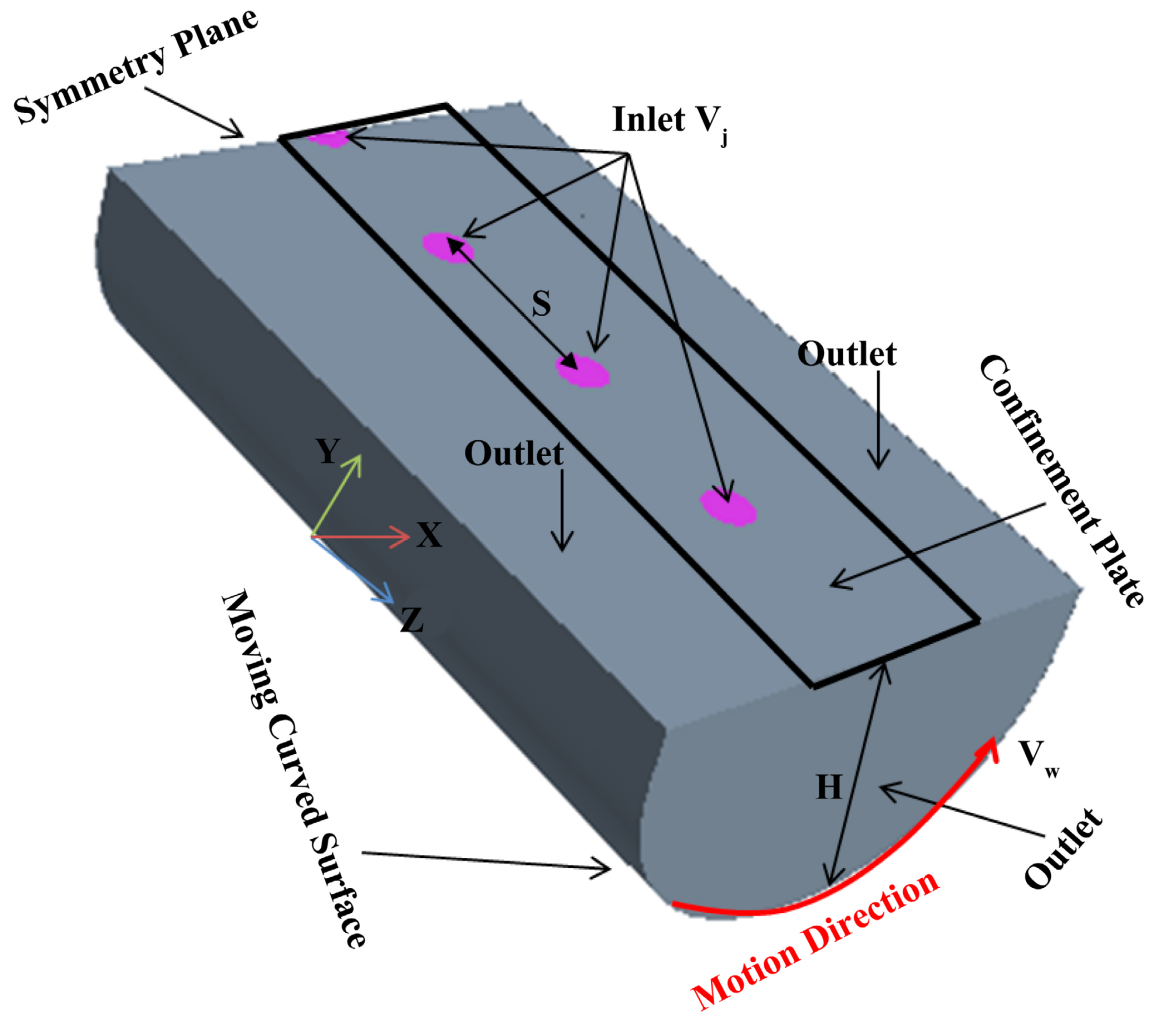

Figure 1. Schematic of the computational domain. 
The final solution was obtained by applying a second-order discretization upwind scheme for the pressure, momentum, and energy terms, and the SIMPLE algorithm is used for pressure-velocity coupling and a segregated flow solver was used for all the calculations. SST $k-\omega$ turbulence model is used because it is recommended as the best compromise between computational cost and accuracy [2] [3]. The flow in the near-wall regime was simulated using a low-Reynolds number approach. The solution was considered to be converged when the value of the scaled residual of the continuity, momentum, and energy equations is less than $10^{-4}$.

An unstructured polyhedral grid was generated using STAR-CCM ${ }^{+}$auto-mesher. Boundary layers with a $y^{+}$value less than one are built on the target surface of the model. The grid was refined near the curved target wall to enable better resolution of the flow in this part. The final numerical model had about 2,157,431 grid cells (see Figure 2).

A grid sensitivity study is carried out to ascertain the accuracy of the numerical results. It is carried out by analyzing the variation of Nusselt number distribution on the target surface along the Z-centerline (the lines pass through the stagnation points of jets). Three simulations with the same geometry but different grid sizes were carried out to check the grid independence of the simulation. The grid sizes are summarized in Table 1 .

The local discretization error distribution is calculated by applying the GCI method [17]. The overall discretization error for fine and intermediate grids was very small $2.6 \%$ and $4.12 \%$ respectively. Therefore, in the present case, the solution

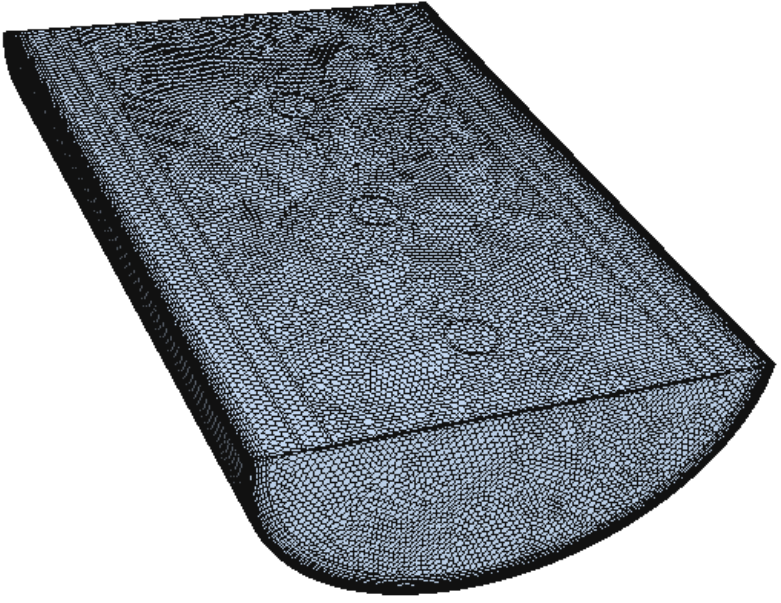

Figure 2. A 3D view of the grid.

Table 1. Grid parameters of the refinement study at $R e=23,000$.

\begin{tabular}{ccccc}
\hline Grid & Base Size $(\mathrm{m})$ & Cell Number & Max $y^{+}$ & Average GCI \% \\
\hline Course & 0.00192 & 447,431 & 0.44 & --- \\
Intermediate & 0.00127 & 970,045 & 0.31 & 4.12 \\
Fine & 0.00088 & $2,157,431$ & 0.23 & 2.6 \\
\hline
\end{tabular}


is considered to be grid-independent. To reduce the computational cost, the intermediate grid is selected as the final grid.

\section{Optimization Method}

In an optimization study, the objectives are evaluated with respect to the design variables using a CFD model. During the analysis, an optimization algorithm uses an embedded strategy to choose the values for the input parameters to best meet the analysis objectives.

The designer should optimize the design parameters of industrial drying equipment to achieve minimum pressure force for force-sensitive products. Therefore, in the present optimization study, the pressure force in the dimensionless form is selected as the objective function to be minimized. Optimization for the multiple jets impinging on a moving curved surface has been performed with respect to five key controlling design parameters as a jet to surface distance, the spacing between the jets, jet exit velocity, surface velocity, and jet exit angle. These parameters can play a critical role in the rate of pressure force and therefore optimization is required for design purposes. Other parameters, such as relative surface curvature $(C r=0.1)$, nozzle diameter $(d=10 \mathrm{~mm})$, jet temperature $\left(100^{\circ} \mathrm{C}\right)$, surface temperature $\left(60^{\circ} \mathrm{C}\right)$, and ambient temperature $\left(20^{\circ} \mathrm{C}\right)$ are held constant. The ranges of the design variables for the optimization have been concentrated on the paper drying machine as shown in Table 2.

The weighted sum of all objectives is used as a numerical optimization method for the pressure force on the moving curved surface to be minimized. The SHERPA search algorithm is used when running an optimization study. If the

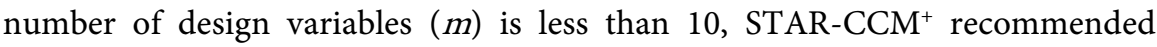
choosing the minimum design number of runs $(N)$ equal to $10 \times m$ to make good progress. Therefore, in the present optimization study, minimum $N$ should be considered equal to 50 . To increase the accuracy of results, $N$ is considered equal to 100 [18].

Weighted sum of all objectives allows an optimization based on a single objective or based on multiple objectives. For the multiple objectives, a linear weighting is used that combines all objectives into a single performance function. Running a

Table 2. Design variables and design space.

\begin{tabular}{ccc}
\hline Design variable & Lower bound & Upper bound \\
\hline$H / d$ & 2 & 10 \\
$S / d$ & 2 & 10 \\
$\theta$ & $40 \mathrm{deg}$ & $90 \mathrm{deg}$ \\
Re number & 4337 & 21,685 \\
Relative surface velocity $(V R)$ & 0.0034 & 1 \\
Inlet velocity & $10 \mathrm{~m} / \mathrm{s}$ & $50 \mathrm{~m} / \mathrm{s}$ \\
Surface velocity & $0.17 \mathrm{~m} / \mathrm{s}$ & $10 \mathrm{~m} / \mathrm{s}$ \\
\hline
\end{tabular}


weighted sum of all objectives analysis with multiple objectives returns a single best design. A design performance is based on the value returned for the objectives together with the degree to which a design satisfies its constraints. Once the constraints are satisfied, only the objectives contribute to the evaluation of performance using the following equation [18]:

$$
\sum_{i=1}^{N_{o b j}} \frac{\operatorname{LinWt}_{i} \cdot \operatorname{Sign}_{i} \cdot \mathrm{Obj}_{i}}{\operatorname{Norm}_{i}}-\sum_{j=1}^{N_{\text {con }}} \frac{\text { QuadWt }_{j} \cdot \text { ConViol }_{j}^{2}}{\text { Norm }_{j}^{2}}
$$

where:

- $N_{o b j}$ is the number of objectives in the design study.

- $L i n W t_{i}$ is the linear weight for the $i$-th objective.

- $\operatorname{Sign}_{i}$ is the sign for the $i$-th objective. The value is -1 for objectives being minimized and +1 for objectives being maximized.

- $O b j_{i}$ is the response value for the $i$-th objective for that design.

- $\mathrm{Norm}_{i}$ is the normalization value for the $i$-th objective.

- $N_{c o n}$ is the number of constraints in the design study.

- QuadWt $t_{j}$ is the quadratic weight for the $j$-th constraint. This value is $10,000.0$.

- Con Viol $l_{j}$ is the amount by which the $j$-th constraint is violated. Con Viol ${ }_{j}$ is 0.0 if the constraint is met.

- $\mathrm{Norm}_{j}$ is the normalization value for the $j$-th constraint, which is the value of the constraint itself. If the constraint value is 0 , then the normalization value is 1 .

The SHERPA algorithm employs multiple search methods simultaneously rather than sequentially. This approach uses the best attributes of each search method. If a particular search method is deemed to be ineffective, this algorithm reduces its participation. While running this algorithm, a combination of global and local search methods is used. At any given time, the number of different methods that are used can range between two and ten. Unlike traditional optimization algorithms that require you to tune parameters manually, the tuning parameters in each method that SHERPA uses are modified automatically during the search. As it learns more about the design space, it determines when and to what extent to use each search method. Therefore, the SHERPA algorithm (Simultaneous Hybrid Exploration that is Robust, Progressive, and Adaptive); a combination of different search methods are used to optimize the efficiency [18].

\section{Results and Discussion}

\subsection{Evaluation of Computational Model}

For numerical predictions of multi-jet impingement heat transfer, a quantification of the numerical accuracy is equally significant. Figure 3 indicates the local Nusselt number distributions along the curvilinear axis on the target surface. The CFD results of this work have been compared with the available data of Fenot et al. (2008) [1] who investigated the heat transfer due to a row of air jets impinging on a fixed concave surface. Uniform heat flux of $4000 \mathrm{~W} / \mathrm{m}^{2}$ was applied 


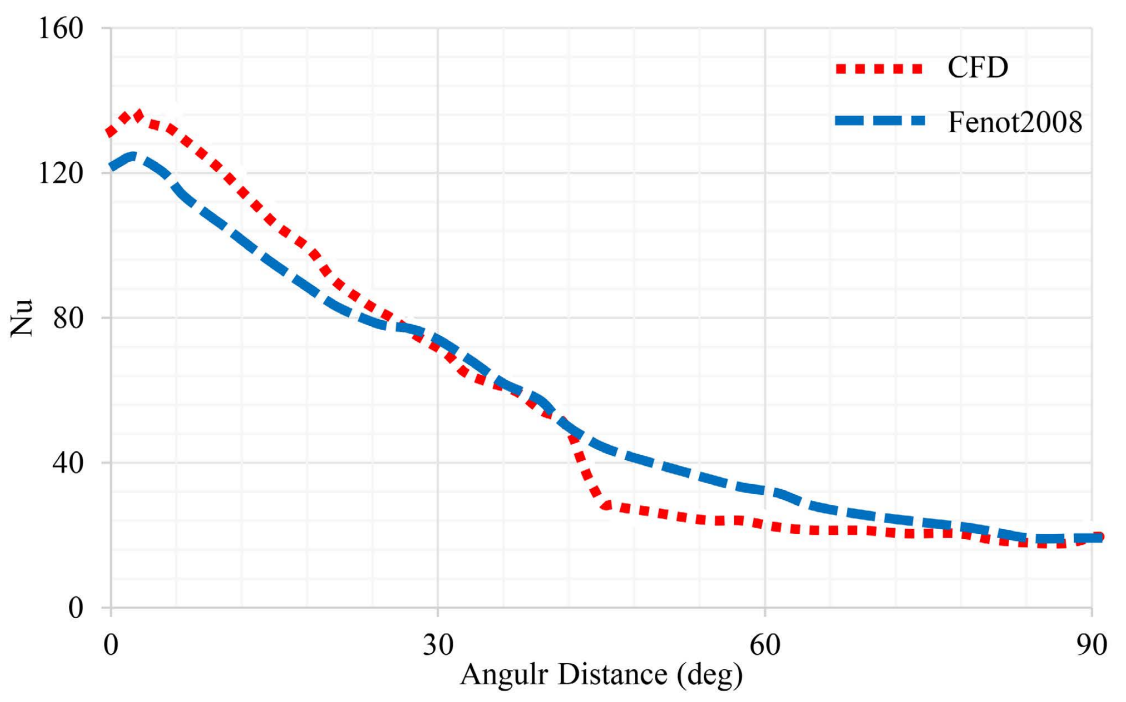

Figure 3. Comparison of the local $\mathrm{Nu}$ distributions along curvilinear axis on fixed curved surface from experiments and CFD $(H / d=5, S / d=4, R e=23,000, \mathrm{Cr}=0.1, \mathrm{VR}=0)$.

to the target surfaces to simulate the condition of the target wall in the experiment. The difference between the experiment and the corresponding values of CFD data is approximately $15 \%$ on average. The agreement between the two is very good and closely followed the same trend as the experimental data. It can be concluded from the evaluation of the turbulence model concerning the predictions of heat transfer that the computational model (discretization, numerical scheme, turbulence model) represents a good compromise between the accuracy of its results and the computational effort.

\subsection{Optimization Results}

\subsubsection{Jet Re Number}

Figure 4 shows the design study with a logarithmic trend line during the numerical optimization for the pressure force coefficient versus Re number.

Increasing the jet $R e$ number occurs with increasing the jet exit velocity from the nozzle and also jet force. Due to the definition of the pressure force coefficient on the impingement surface (see Equation (8)), the pressure force coefficient is relatively insensitive to the jet Reynolds number within the range examined. This result correlates with the findings of Wang et al. [11] for the single impinging jet on a fixed flat surface.

\subsubsection{Nozzle to Surface Distance}

Figure 5 shows the design study with a logarithmic trend line during the numerical optimization for the pressure force coefficient versus nozzle to surface distance $(H / d)$.

The pressure force coefficients on the target surface are highly dependent on the nozzle to surface distance and the pressure force coefficients increase with decreasing the $H / d$ due to the decrease in the momentum exchange between the jet flow and the ambient leads to the increase in the pressure on the impingement 


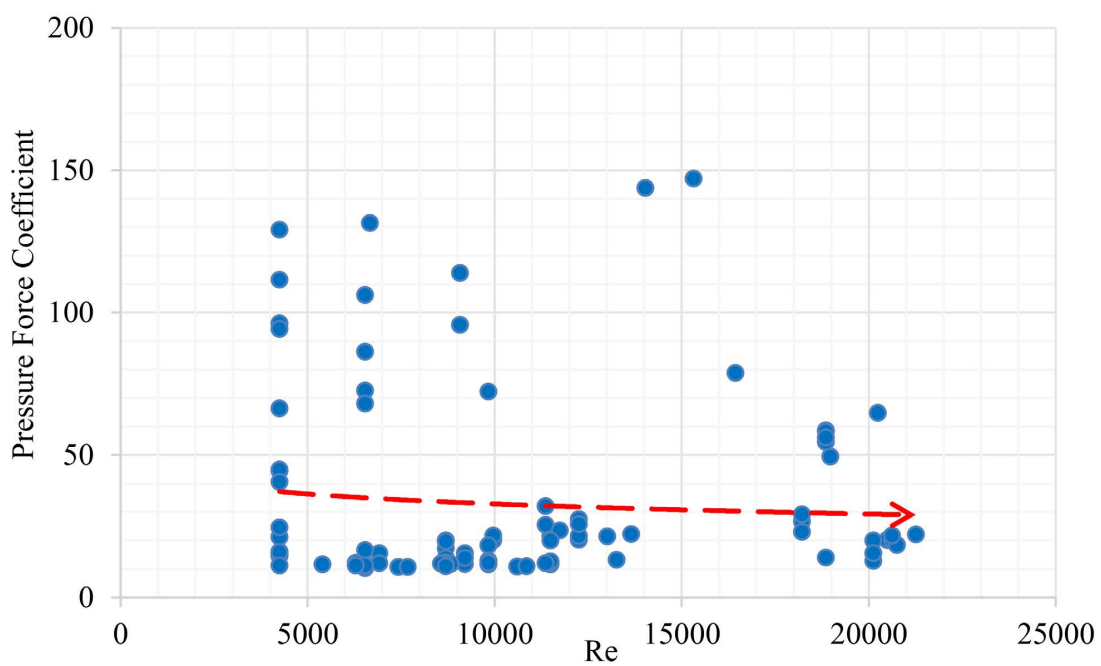

Figure 4. Design study during the numerical optimization for the pressure force coefficient versus $R e$.

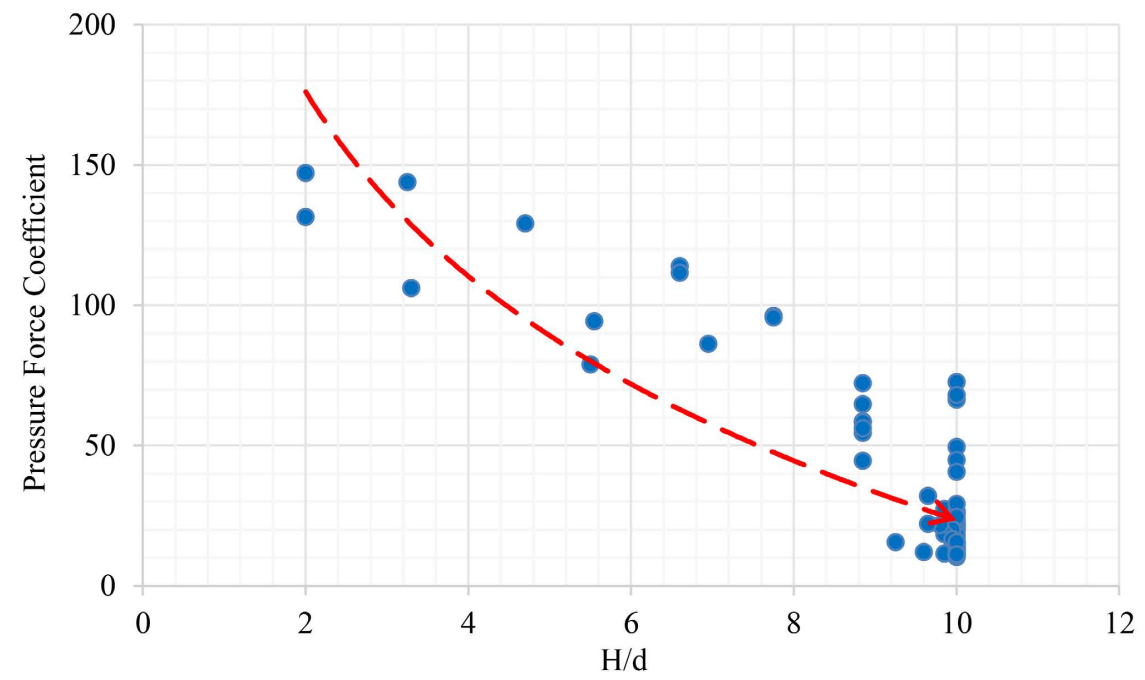

Figure 5. Design study during the numerical optimization for the pressure force coefficient versus $H / d$.

surface. Therefore, for products sensitive to the pressure force, a high value of $H / d$ is advisable (see Figure 6). This result coincides with the findings of Wang et al. [11] for the single impinging jet on a fixed flat surface.

\subsubsection{Jet to Jet Spacing}

Figure 7 shows the design study with a logarithmic trend line during the numerical optimization for the pressure force coefficient versus jet to jet spacing $(S / d)$. Results indicate that the pressure force coefficients on the impingement surface are relatively insensitive to the jet to jet spacing $(S / d)$ within the range examined.

\subsubsection{Jet Angle}

Figure 8 shows the design study with a logarithmic trend line during the numerical 


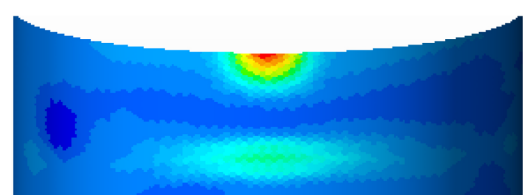

$H=3 d$

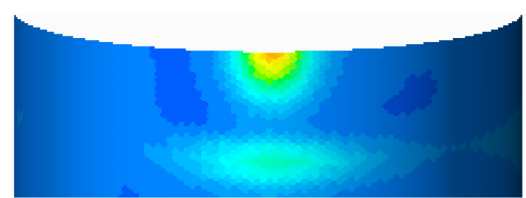

$\mathrm{H}=5 \mathrm{~d}$

Pressure $(\mathrm{Pa})$

$-62.00$

117.20

296.40

475.60

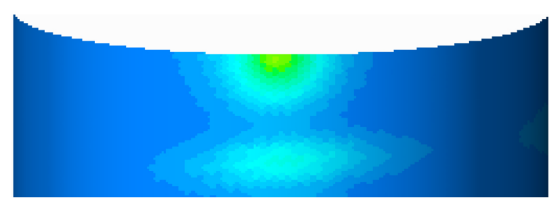

$H=10 d$

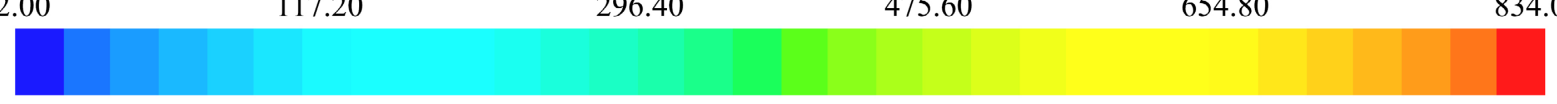

Figure 6. Pressure distribution from central jet impinging on a moving curved surface for the different nozzle to surface distance $(R e=23,000, S / d=4, V R=0.28$, and $\theta=90 \mathrm{deg})$.

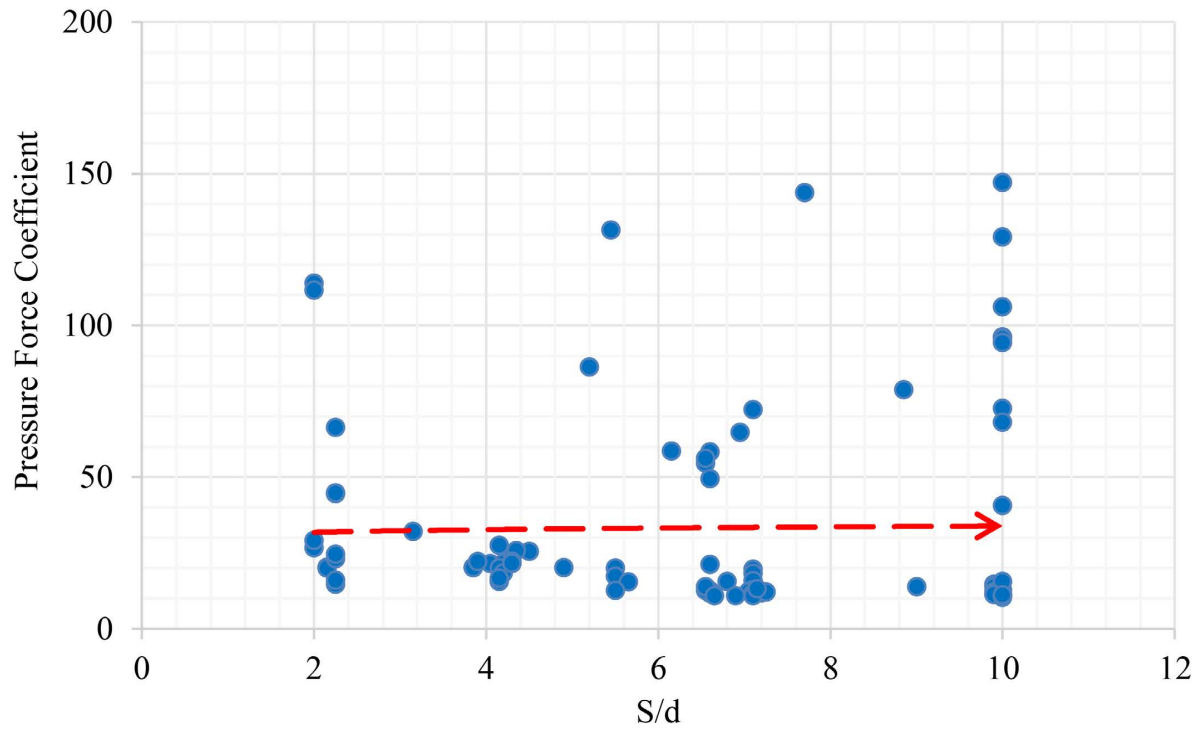

Figure 7. Design study during the numerical optimization for the pressure force coefficient versus $S / d$.

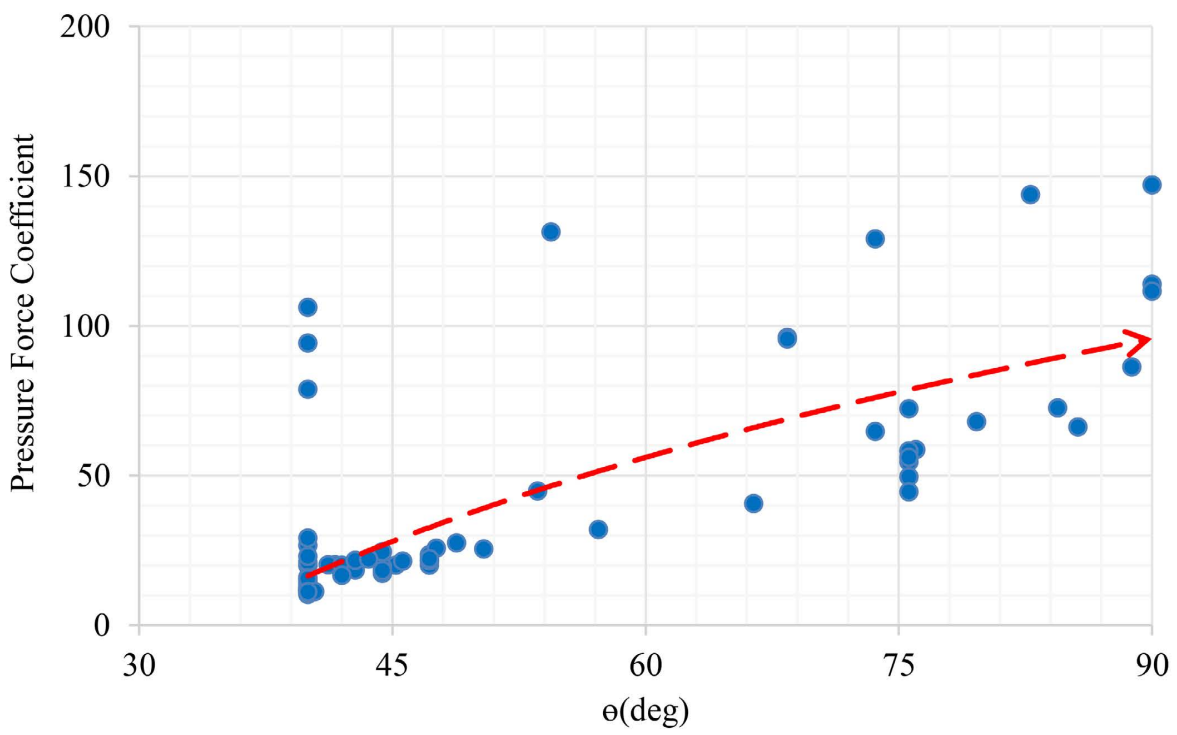

Figure 8. Design study during the numerical optimization for the pressure force coefficient versus $\theta$. 
optimization for the pressure force coefficient versus jet angle $(\theta)$. The jet angle is varied between $40^{\circ}$ and $90^{\circ}$ as measured with respect to the horizontal axes and the case of $90^{\circ}$ corresponds to the orthogonal jet with maximum pressure force.

The pressure force coefficient correlates strongly with the orthogonal component of the jet flow. With decreasing the jet angle, the orthogonal component of the jet flow decreases but on the other hand, the flow component parallel to the wall increases having the opposite effect. Therefore, the pressure force coefficient is highly dependent on the jet angle $(\theta)$ and increases with increasing the jet angle. This is to be expected because when the jet is directed orthogonal to the surface (90 deg); it can exert the most pressure upon striking the surface (see Figure 9). This result coincides with the findings of Peper et al. [16] for radial jets impinging on a fixed flat surface.

\subsubsection{Relative Surface Velocity}

Figure 10 shows the design study with a logarithmic trend line during the numerical optimization for the pressure force coefficient versus relative surface

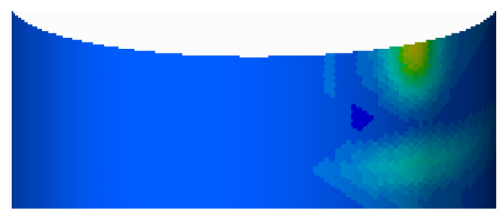

45 deg

$-32.00$

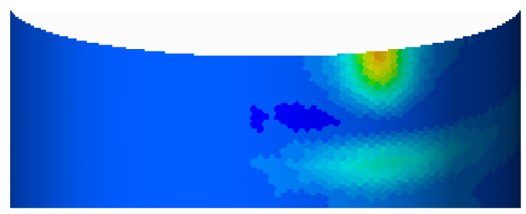

$60 \mathrm{deg}$

Pressure $(\mathrm{Pa})$

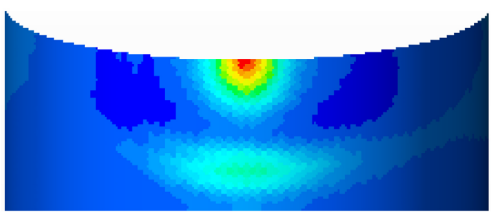

$90 \mathrm{deg}$

558.40

776.00

Figure 9. Pressure distribution from central jet impinging on a moving curved surface for different jet angle $(R e=23,000, H / d$ $=1, S / d=4$, and $V R=0.28$ ).

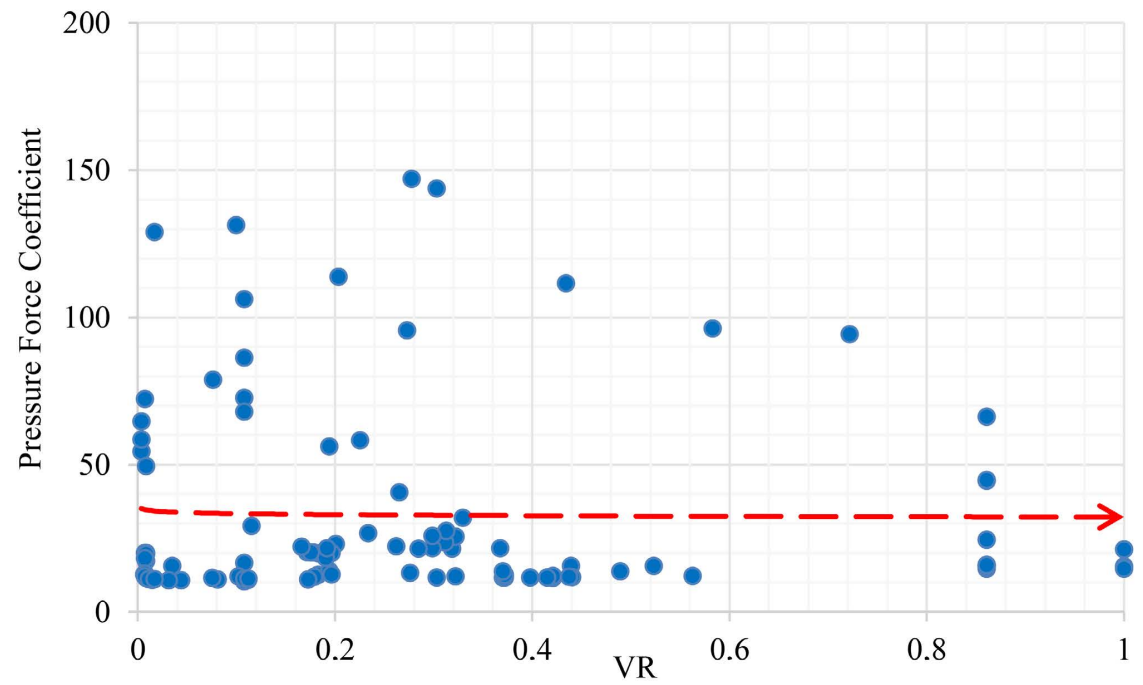

Figure 10. Design study during the numerical optimization for the pressure force coefficient versus $V R$. 
velocity $(V R)$. Results indicate that the pressure force coefficients on the impingement surface are relatively insensitive to the relative surface velocity within the range examined.

Table 3 shows a summary of results during the numerical optimization. It can be concluded from the above figures that the pressure force coefficient is highly dependent on the nozzle to surface distance $(H / d)$ and jet angle $(\theta)$ but relatively insensitive to jet inlet velocity $\left(V_{j}\right)$, jet to jet spacing $(S / d)$, and surface velocity $\left(V_{w}\right)$. The minimum pressure force coefficient correlates with a high value of nozzle to surface distance and a low value of the jet angle. However, the best design in this study is found at $H / d=10, S / d=10, \theta=40^{\circ}, V_{j}=15.37 \mathrm{~m} / \mathrm{s}$ and $V_{w}$ $=1.66 \mathrm{~m} / \mathrm{s}$ for the investigated parameters in the range of $H / d=2-10, S / d=2$ 10, $\theta=40^{\circ}-90^{\circ}, V_{j}=10-50 \mathrm{~m} / \mathrm{s}$ and $V_{w}=0.17-10 \mathrm{~m} / \mathrm{s}$ under $d=10 \mathrm{~mm}$ as shown in Table 3. Generally, the best designs are in the range of $H / d=9.85-10$, $S / d=6.65-10, \theta=40^{\circ}, V_{j}=10-25.5 \mathrm{~m} / \mathrm{s}$ and $V_{w}=0.17-8.6 \mathrm{~m} / \mathrm{s}$. There were no appropriate results reported in the literature for comparison with the present results for the pressure coefficient.

\section{Correlation Equation}

One correlation for the pressure force coefficient is developed for the single row of jets impinging on a moving curved surface. A multiple regression fit is applied for the development of this correlation equation from the numerical simulation (hundred design points during the numerical optimization) and the $R^{2}$ value is 0.97 :

$$
C_{f}=120.2 R e^{0.09}(H / d)^{-0.72}(S / d)^{-0.37} \theta_{r a d}^{1.96}(V R)^{-0.008}
$$

Table 3. Results during the numerical optimization.

\begin{tabular}{cccccc}
\hline Objectives & $H / d$ & $S / d$ & $\theta(\mathrm{deg})$ & $V_{j}(\mathrm{~m} / \mathrm{s})$ & $V_{w}(\mathrm{~m} / \mathrm{s})$ \\
\hline $\operatorname{Min} C_{f}$ & 10 & 10 & 40 & 15.37 & 1.66 \\
\hline
\end{tabular}

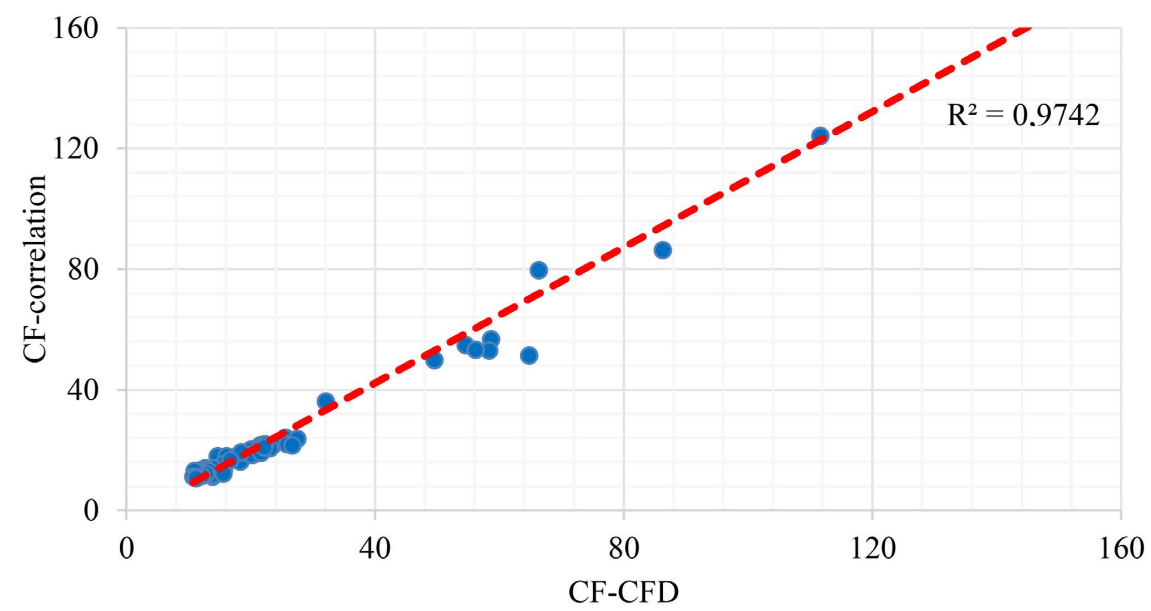

Figure 11. Parity plot showing a comparison between the force coefficient predicted by the CFD and correlation. 
The above correlation is proposed in terms of $R e, H / d, S / d, \theta$, and $V R$ as the independent variables for $R e$ number in the range of 4337 to $21,685, H / d$ from 2 to $10, S / d$ from 2 to $10, \theta$ from $40^{\circ}$ to $90^{\circ}$ and $V R$ from 0.0034 to 1 . The observed trends in numerical simulations are shown by the exponents of the independent parameters in the correlation. The agreement in the prediction of the pressure force coefficient between the numerical simulation and correlation is found to be reasonable and all the data points deviate from the correlation approximately $8 \%$ on average (see Figure 11).

\section{Conclusions}

The numerical simulations and optimization of multiple circular jets impinging on a moving curved surface are carried out and the commercial CFD package STAR $\mathrm{CCM}^{+}$is employed with the SST $k$ - $\omega$ turbulence model to simulate a real geometry for industrial drying applications. The designer should optimize the design parameters of industrial drying equipment to achieve the minimum pressure force for force-sensitive products or surfaces.

The optimization has been performed with respect to five design parameters as a jet to surface distance, the spacing between the jets, jet inlet velocity, surface velocity, and jet angle. The jet inlet velocity has a strong influence on the pressure force, but due to its definition a negligible influence on the pressure force coefficient. As the orthogonal jet component determines the pressure force, the jet angle is influential. The nozzle to surface distance is also an important design parameter. The jet to jet spacing and the surface velocity are of much lesser importance with regard to the pressure force coefficient.

For the optimization of the impinging jet, the pressure force on the moving curved surface is set as the objective function to be minimized. Hundred design points are selected by the SHERPA search algorithm within the design space. The minimum values of the pressure coefficients were found at the margins of the design space with regard to angle and surface distance.

One correlation describing the pressure force has been developed and validated. The agreement in the prediction of the pressure force between the numerical simulation and correlation is found to be reasonable. The results of this research lead to a significant reduction in the pressure force on force-sensitive products or surfaces in industrial drying applications.

\section{Conflicts of Interest}

The authors declare no conflicts of interest regarding the publication of this paper.

\section{References}

[1] Fenot, M., Dorignac, E. and Vullierme J.-J. (2008) An Experimental Study on Hot Round Jets Impinging a Concave Surface. International Journal of Heat and Fluid Flow, 29, 945-956. https://doi.org/10.1016/j.ijheatfluidflow.2008.03.015 
[2] Kadiyala, P.K. and Chattopadhyay, H. (2017) Numerical Simulation of Transport Phenomena Due to Array of Round Jets Impinging on Hot Moving Surface. Drying Technology, 35, 1742-1754. https://doi.org/10.1080/07373937.2016.1275672

[3] Heo, M.W., Lee, K.D. and Kim, K.Y. (2012) Parametric Study and Optimization of Staggered Inclined Impinging Jets on a Concave Surface for Heat Transfer Augmentation. Numerical Heat Transfer, Part A: Applications, 61, 442-462. https://doi.org/10.1080/10407782.2012.654453

[4] Penumadu, P.S. and Rao, A.G. (2017) Numerical Investigations of Heat Transfer and Pressure Drop Characteristics in Multiple Jet Impingement System. Applied Thermal Engineering, 110, 1511-1524.

https://doi.org/10.1016/j.applthermaleng.2016.09.057

[5] Hebert, R.T., Ekkad, S.V., Gao, L. and Bunker, R.S. (2005) Impingement Heat Transfer, Part II: Effect of Streamwise Pressure Gradient. Journal of Thermophysics and Heat Transfer, 19, 66-71. https://doi.org/10.2514/1.8588

[6] El-Gabry, L.A. and Kaminski, D.A. (2005) Numerical Investigation of Jet Impingement with Crossflow-Comparison of Yang-Shih and Standard k- $\epsilon$ Turbulence Models. Numerical Heat Transfer, Part A: Applications, 47, 441-469. https://doi.org/10.1080/10407780590891254

[7] Kamal, R.M., El sayed Mostafa, M. and Abdel Aziz, S.S. (2006, December) An Experimental Study of an Oblique Multiple Circular Air Jets Impingement on a Flat Plate. 8 th International Congress of Fluid Dynamics and Propulsion, Sharm El-Sheikh, 14-17 December 2006.

[8] Harrington, J., Hossain, J., Wang, W., Kapat, J., Maurer, M. and Thorpe, S. (2017) Effect of Target Wall Curvature on Heat Transfer and Pressure Loss from Jet Array Impingement. Journal of Turbomachinery, 139, Article ID: 051004.

https://doi.org/10.1115/1.4035160

[9] Levy, Y., Rao, A.G., Erenburg, V., Sherbaum, V., Gaissinski, I. and Krapp, V. (2012, June) Pressure Losses for Jet Array Impingement with Crossflow. ASME Turbo EXpo 2012: Turbine Technical Conference and Exposition. Volume 4: Heat Transfer, Parts $A$ and B, Copenhagen, 11-15 June 2012, 139-149. https://doi.org/10.1115/GT2012-68386

[10] Kastner, W. and Rippel, R. (1988) Jet Impingement Forces on Structures-Experiments and Empirical Calculation Methods. Nuclear Engineering and Design, 105, 269-284. https://doi.org/10.1016/0029-5493(88)90249-X

[11] Wang, X.K., Niu, G.P., Yuan, S.Q., Zheng, J.X. and Tan, S.K. (2015) Experimental Investigation on the Mean Flow Field and Impact Force of a Semi-Confined Round Impinging Jet. Fluid Dynamics Research, 47, Article ID: 025501. https://doi.org/10.1088/0169-5983/47/2/025501

[12] Page, R.H., Hadden, L.L. and Ostowari, C. (1989) Theory for Radialjet Reattachment Flow. AIAA Journal, 27, 1500-1505. https://doi.org/10.2514/3.10294

[13] Page, R.H., Carbone, J. and Ostowari, C. (1990) Radial Jet Reattachment Force. EXperiments in Fluids, 8, 297-298. https://doi.org/10.1007/BF00187233

[14] Thiele, E.W., Dautel, S.W., Page, R.H. and Seyed-Yagoobi, J. (1993) A New Paper Machine Drying Concept: A Multifunctional, Radial-Jet Reattachment Nozzle Blow Box. TAPPI Papermakers Conference, 76, 203-207.

[15] Thiele, E.W., Seyed-Yagoobi, J., Page, R.H. and Castillo-Garcia, H. (1995) Enhancement of Drying Rate, Moisture Profiling and Sheet Stability on an Existing Paper Machine with RJR Blow Boxes. TAPPI Papermakers Conference, Chicago, 23-26 April 1995, 223-228. 
[16] Peper, F., Leiner, W. and Fiebig, M. (1997) Impinging Radial and Inline Jets: A Comparison with Regard to Heat Transfer, Wall Pressure Distribution, and Pressure Loss. Experimental Thermal and Fluid Science, 14, 194-204.

https://doi.org/10.1016/S0894-1777(96)00066-0

[17] Roache, P.J. (2003) Conservatism of the Grid Convergence Index in Finite Volume Computations on Steady-State Fluid Flow and Heat Transfer. Journal of Fluids Engineering, 125, 731-735. https://doi.org/10.1115/1.1588692

[18] Siemens PLM. STAR-CCM+. https://www.plm.automation.siemens.com/ 


\section{Nomenclature}

$A_{f}$ open area ratio, total jet area to heat transfer area

$A$ : surface area $\left(\mathrm{m}^{2}\right)$

$C_{f}$ force coefficient

Con Viol; : violated amount by $j$-th constraint

$d$ jet exit diameter $(\mathrm{m})$

$F$ : pressure force $(\mathrm{N})$

$h$ : heat transfer coefficient $\left(\mathrm{W} / \mathrm{m}^{2} \mathrm{~K}\right)$

$H$ : nozzle to surface distance $(\mathrm{m})$

$k$ : thermal conductivity $(\mathrm{W} / \mathrm{mK})$

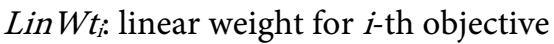

$m$ : number of design variables

$N$ : number of runs

$N_{\text {con }}$ : number of constraints

$N_{o b j}$ number of objectives

Norm $_{i}$ : normalization value for $i$-th objective

Norm $_{j}$ : normalization value for $j$-th constraint

$N u$ : Nusselt number

Obj: i-th objective

$P$ : pressure $(\mathrm{Pa})$

$q$ : convective heat flux $\left(\mathrm{W} / \mathrm{m}^{2}\right)$

Quad $W t_{j}$ : quadratic weight for $j$-th constraint

Re: Reynolds number

$S$ : jet to jet spacing $(\mathrm{m})$

$\operatorname{Sign}_{i}$ : sign for $i$-th objective

T: temperature $(\mathrm{K})$

$T_{i:}$ Reynolds stress tensor

$U:$ velocity $(\mathrm{m} / \mathrm{s})$

$\bar{U}$ : average velocity $(\mathrm{m} / \mathrm{s})$

$U_{i:}$ instantaneous components of the velocity vector in the direction $X_{i}(\mathrm{~m} / \mathrm{s})$

$U_{i}^{\prime}$ : fluctuating components of the velocity vector in the direction $X_{i}(\mathrm{~m} / \mathrm{s})$

$X, Y, Z$ : coordinates

$V$ : jet exit velocity $(\mathrm{m} / \mathrm{s})$

$y^{+}$: dimensionless wall distance

\section{Greek Letters}

$k$ turbulence kinetic energy $\left(\mathrm{m}^{2} \cdot \mathrm{s}^{-2}\right)$

v: kinematic viscosity $\left(\mathrm{m}^{2} / \mathrm{s}\right)$

$v_{t}$ : turbulent viscosity $\left(\mathrm{m}^{2} / \mathrm{s}\right)$

$\omega$ : specific dissipation rate of turbulence kinetic energy (1/s)

$\theta$. jet inclined angle with respect to the horizontal axes (deg)

$\rho$ : density $\left(\mathrm{kg} / \mathrm{m}^{3}\right)$

$\delta_{i j}$ : Kronecker delta 
$S_{i j}$ : mean strain rate tensor $\left(\mathrm{m} / \mathrm{s}^{2}\right)$

$\Theta$ : general scalar variable

$\Gamma_{\Theta}$ : diffusivity of $\Theta\left(\mathrm{m}^{2} / \mathrm{s}\right)$

\section{Subscripts}

ave: average

j: jet

opt: optimum

st: stagnation point

w: wall

\section{Abbreviation}

Cr. curvature ratio; nozzle to surface diameter CFD: computational fluid dynamic

GCI: grid convergence index

$V R$ : velocity ratio; surface to jet velocity

SST: shear stress transport 